\title{
The immunological impact of orthomolecular medicine using bioactive compounds as key factors in endometriosis
}

\author{
Alexandros Vlachos ${ }^{1}$ and Simon Vassiliadis ${ }^{2}$ \\ ${ }^{1}$ Department of Pediatric Surgery, Penteli General Children's Hospital, Palaia Penteli, Athens, \\ 15236, Greece; ${ }^{2}$ Association of Greek Immunology Graduates, Maroussi, Athens, 15125, \\ Greece
}

Corresponding Author: Simon Vassiliadis, Ph.D. Principal Investigator. Association of Greek Immunology Graduates, 33 Voriou Ipirou Street, Maroussi, 15125 Athens, Greece.

Submission date: August $10^{\text {th }}, 2018$, Acceptance Date: January $28^{\text {th }}, 2019$, Publication Date: January $31^{\text {st }}, 2019$

Citation: Vlachos A., Vassiliadis S. The immunological impact of orthomolecular medicine using bioactive compounds as key factors in endometriosis. Bioactive Compounds in Health and Disease 2019; 2(1): 1-10. DOI: https://doi.org/10.31989/bchd.v2i1.555

\begin{abstract}
Endometriosis, an inflammatory, non-lethal, non-malignant disease, still has unjustified etiology. Among many, the theory dealing with this review claims that a suppressed or incompetent immune system that is totally unable to eradicate the non-hemopoietic mesenchymal endometriotic stem cell (MESC) escapes immune surveillance. As a result, there is migration and invasion of the aforementioned cell to ectopic tissues causing the disease.

This review focuses on bioactive compounds (i.e. vitamins and minerals) that may have the potential to boost the immune system rendering it capable to fight the MESC and, consequently, endometriosis. The use of vitamins and minerals, also called meganutrients, constitutes the known approach of orthomolecular medicine. However, when scrutinized these methods yield contradicting results but still merit attention. According to our review of the available scientific literature, compared to traditional medicine, which only regulates hormonal imbalances and alleviates pain, the orthomolecular approach is promising for successfully strengthening the immune system towards a curative outcome. However, the underlying mechanisms behind the action of the abovementioned natural bioactive ingredients appear numerous and often speculative, thereby remaining under examination.
\end{abstract}

Keywords: bioactive compounds, functional foods, endometriosis, orthomolecular medicine, mesenchymal endometriotic stem cell (MESC), immune system

\section{INTRODUCTION}

Endometriosis, from the ancient Greek endo= inside, metrawomb and iosisdisease due to a virus, is an inflammatory, non-lethal, and non-malignant disease affecting approximately $10 \%$ to $15 \%$ of all women of reproductive age [1] and $70 \%$ of women with chronic pelvic pain [2]. Endometriosis is defined as the presence of endometrial glands and stroma-like lesions outside of the uterus (ectopic endometrial implants) and associated with infertility and pelvic pain [1]. 
However, it is unknown whether infertility is due to the endometriotic state of women or whether other endometriosis-associated factors lead to infertility. Mechanisms proposed for the development of infertility in endometriotic women include a number of gynecologic reasons, already extensively described, in addition to other causes that include the use of chemicals, Candida albicans infection, or abnormalities [3-6]. Numerous reports present evidence that endometriosis is a genetic and pre-determined disease due to environmental, endocrinological, and immunological factors [5, 7-10]. However, the discovery and investigation of a non-hemopoietic mesenchymal stem cell (MESC) [11] has shown that MESC(s) can escape surveillance due a frail immune system not competent enough to intercept the immaturely senescent cell, allowing migration and invasion to ectopic tissues [12, 13]. Thus, certain constituents of foods, termed bioactive compounds, can provide beneficial health properties, such as anti-inflammatory and anti-fungal activity, antioxidant advantages and especially preventive action against disease [14]. These bioactive compounds have recently been defined by Dr. Martirosyan and Pisarski as "primary and secondary metabolites of nutritive and non-nutritive natural components generating health benefits by preventing or managing chronic disease or its symptoms" [14-15].

Furthermore, the Functional Food Center (https://www.functionalfoodscenter.net/) defines "functional foods" as "natural or processed foods that contain biologically-active compounds; which, in defined, effective non-toxic amounts, provide a clinically proven and documented health benefit utilizing specific biomarkers, for the prevention, management, or treatment of chronic disease or its symptoms". Nevertheless, while many studies have been published using bioactive compounds in defined and non-toxic amounts for various conditions, accessible information on endometriosis is particularly lacking. As a result, we attempted to succinctly present the action of beneficial endometriosis substances as a function of their dosage and effect. The use of meganutrients (vitamins and minerals), constituting the orthomolecular approach, has been shown to be successful when taken up by endometriotic subjects, as they are able to reduce pain, ease the disease via anti-inflammatory effects, and promote immunity, which may be a key factor in intercepting the MESC for preventing or correcting endometriosis.

\section{THE CONCEPT OF ORTHOMOLECULAR MEDICINE AND ITS ACTION ON IMMUNE PARAMETERS RELATING TO ENDOMETRIOSIS}

The term "orthomolecular" was first used by the two-time Nobel Laureate Linus Pauling in 1968 to characterize the treatment of disease with nutrients that are endogenous to the human body [16]. Orthomolecular, from the Greek ortho=correct, basically translates into "essential nutrient". Orthomolecular physicians treat disease by varying dosages of "correct molecules" that are required but not synthesized by the human body. Orthomolecular therapy can be preventive, protective, and/or corrective. Its practitioners prevent disease by helping patients against the dangerously polluted environment and nutrient-stripped refined foods. With the uptake of a megavitamin therapy and/or a load of meganutrients. For example, with calcium, vitamin $\mathrm{C}$, and zinc patients may be protected from the harmful effects of lead, cadmium, and mercury. In fact, orthomolecular medicine began and still focuses on the correction of biochemical imbalances of disease and represents another form of alternative medicine aiming to maintain human health through nutritional supplementation [17]. The first megavitamin therapy report, as presented by Andrew Saul, was published back in 1935 when Claus Washington Jungeblut, MD, Professor of Bacteriology at Columbia University first published on vitamin $\mathrm{C}$ as prevention and treatment for polio [18]. In the same year, he also demonstrated that vitamin $\mathrm{C}$ could inactivate diphtheria toxin, 
while the first report on endometriosis came out in 1940 by the Shute brothers revealing that vitamin E prevents endometriosis [18].

With birthing problems and infant mortality, the use of certain vitamins and supplements has well been documented [19]. Similarly, for endometriosis a number of vitamins, supplements and/or trace elements can be mentioned as their role on endometriosis. As a whole, they have been described (i.e. on endometriotic lesions, fertility, pain, hormonal and/or immune regulation). As discussed below, their beneficial action has been well documented in endometriosis and to immunological fortification of the organism. However, the enhancement of immunity observed has never been correlated to the induction of the proper conditions for the interception of the MESC at early stages, thereby to reverse the burden of the disease.

Accordingly, a thorough literature search has revealed the following results on endometriosis in relation to bioactive compounds:

- Omega -3 fatty acids in fish oil are known to improve immunity and have antiinflammatory properties. They are especially important for endometriotic women as they increase the body's production of prostaglandin E1 (PGE1), helping to manage painful symptoms. Additionally, a 2013 study by Herington et al. using an experimental mouse model of endometriosis, demonstrated that in mice with a $10 \%$ supplemented fish oil diet, adhesion score and disease burden significantly reduced [20].

- Melatonin has anti-cancer and anti-inflammatory properties as studied by Güney et al., in a mouse model of endometriosis [21]. The authors found that melatonin causes regression and atrophy of the endometriotic lesions in experimental mice.

- Medicinal cannabis: As endometriosis is a benign disease usually responding to natural substances with anti-cancer activity, one would expect Cannabis sativa extract ( $\beta$ caryophyllene constituent of essential oil of Cannabis sativa) to have beneficial effects in endometriotic subjects. Indeed, suppression of the growth of endometriotic implants by $52.5 \%$ compared with controls when used at $10 \mathrm{mg} / \mathrm{kg}$ was demonstrated. Additionally, $\beta$-caryophyllene produced apoptosis in luminal epithelium of the cyst, as well as in endothelial cells of blood vessels [22]. An additional benefit, reduction in endometriosis-associated pain, was also reported [23].

- Diindolylmethane (DIM) supports the body in eliminating excess estrogen. DIM is derived from cruciferous vegetables like cauliflower and broccoli. It metabolizes estrogen into components easily assimilated and removed by the body and avoids the development of estrogen dominance [24, 25].

- Coenzyme Q10 (CoQ10) is a potential antioxidant and immune support. However, studied on endometriosis are lacking [26, 27].

- Resveratrol is a natural substance obtained from red wine that has anti-proliferative and anti-inflammatory properties [28]. The mechanisms/pathways of action of this naturally occurring compound have also shown that, in animal models of endometriosis, resveratrol supplementation displays beneficial results by decreasing the number and volume of endometrial implants, suppressing proliferation, vascularization, 
inflammation, cell survival, and increasing apoptosis [29]. On the other hand, in vitro resveratrol treatment reduces invasiveness of endometriotic stromal cells (ESCs) and suppresses their inflammatory responses. [29]. A 2011 study by Bruner-Tran et al. has demonstrated that resveratrol inhibits development of experimental endometriosis in vivo and reduces endometrial stromal cell invasiveness in vitro [30]. In this study, nude mice were implanted with human endometriosis cells. The authors found that resveratrol decreased the number of endometrial implants by $60 \%$ and decreased total volume of implants by $80 \%$, a quite impressive result for a naturally occurring supplement. Finally, in a 2017 randomized clinical trial, resveratrol at $40 \mathrm{mg} / \mathrm{d}$ given with monophasic contraceptive pill has not been found superior to placebo for treatment of pain in endometriosis [31].

- L-Carnitine, naturally produced in the human body and helping the body convert fat to energy, appears allegedly useful in endometriosis since it facilitates pregnancy by reducing embryo cell death and damage to oocytes or eggs in endometriosis-infertile women who are trying to get pregnant or undergoing in vitro fertilization [32]. However, when administered to young female mice, L-Carnitine has been shown to induce a pathological condition resembling stage IV human endometriosis accompanied by a marked degree of infertility. Accordingly, the use of this nutrient by young women may be potentially hazardous responsible for the onset of endometriosis at a later stage of their lives [33].

- Iron (found in clams, fortified cereals, liver, oysters, lean red meat and dried beans) prevents anemia, weakness and fatigue due to heavy bleeding. Women with endometriosis tend to have very heavy periods that can lead to an iron deficiency. This can cause anemia, which is characterized by extreme fatigue and weakness [34].

- Magnesium (abundant in bananas, barley, green beans, kelp, sunflower seeds, and raspberry leaves) is extremely important, as it is a mineral essential for activating chemical reactions, transporting glucose to the cells, and boosting the immune system. Magnesium is also a muscle relaxant decreasing cramping [35].

- Selenium (cabbage, celery, cucumbers, Brazil nuts, tuna, cod, and meats) has been historically given to cows by farmers to prevent endometriosis. Endometriosis hinders fertility, so farmers work hard to prevent a disease that results in fewer calves. Selenium boosts immune system; decreases inflammation associated with endometriosis when taken with vitamin E [36]. This mineral increases natural killer cells and mobilizes cancer-fighting cells. A supplement of selenium is advised for women with endometriosis. The best sources of selenium may be from plant foods. However, most soil is depleted in this trace element today, so plants are not able to take up this mineral [34].

- Zinc (in ginger root, oysters, lamb chops, and pecans) is critical for proper thymus gland and immune system function. Research has shown that daily intake of $30 \mathrm{mg}$ of zinc reactivates the immune system with dramatic improvements after 6 months in those with zinc deficiency. This valuable mineral increases the production of white blood 
cells that fight infection and helps them fight more aggressively. It also increases killer cells that fight against cancer and helps the release of antibodies. Zinc supplements have been shown to slow the growth of cancer. However, too much zinc in the form of supplements (more than 75 milligrams a day) can inhibit immune function [37].

- Vitamin A (found in apricots, broccoli, cantaloupe, carrots, eggs, milk, pumpkin, spinach, and squash) improves the immune system. Vitamin A is an antioxidant and helps lessen profuse menstrual bleeding [35].

- Vitamin B complex (in fortified cereals, beans, red meat, poultry, mollusks, and liver) helps break down proteins, carbohydrates, and fats in the body, helps keep estrogen levels naturally low, and helps produce good PGs. Women with endometriosis may produce high levels of PGE2, resulting in inflammation in tissues and painful cramping. One of the B vitamins, B6, has been shown to significantly reduce the intensity and duration of period pains, thereby helping many sufferers [38]. B vitamins are also crucial for the conversion of essential fatty acids into beneficial PGs having a relaxing effect on the womb muscles and anti-inflammatory properties [37].

- Vitamin C (berries, broccoli, cantaloupe, grapefruit, lemons, oranges, peppers, spinach, and strawberries) helps boost the immune system and fight off disease. Vitamin $\mathrm{C}$ is an antioxidant that helps control excessive bleeding and detoxifies pollutants [34].

- Vitamin D (found in fatty fish, such as tuna, mackerel, salmon, and fish oils; however, the main source of this vitamin is still sunlight exposure) is an important player in the regulation of inflammation, as its deficiency enhances inflammation and promotes immunosuppression through the expansion of $\mathrm{T}$ regulatory cells (Tregs) in a murine myometrium (the middle layer of uterus, in contact with the endometrium) model [39]. As established, tumor escapement from immune response is due to an immunosuppressive environment, thereby pointing to a potential relationship with the inability of the immune system to annihilate the MESC, since endometriosis accurately resembles a cancerous state without being lethal [40].

- Vitamin E (abundant in almonds, avocado, eggs, safflower oil, salmon, and sunflower oil) is known to ensure that animals have healthy uterine linings and used by farmers since the 1930's. Vitamin E improves the immune system and has been shown to relieve menstrual cramps in 70 percent of women within two menstrual cycles in endometriosis

- Beta carotene (in carrots, spinach, sweet potatoes, tomatoes, and cantaloupe) increases the number of infection-fighting cells, natural killer cells, and helper T-cells. Beta carotene is also a powerful antioxidant that mops up excess free radicals that accelerate ageing and converts to vitamin $\mathrm{A}$ in the body [41].

- Probiotic supplements (containing Lactobacillus acidophilus): a healthy microbiome is essential for the management of endometriosis. There is a community of gut bacteria and specifically bacterial genes, called the estrobolome, that produce an enzyme supporting estrogen metabolization. Thus, probiotics can also be recommended for 
maintenance of gastrointestinal and immune health. However, people who are severely immunocompromised or taking immune suppressive drugs should speak to their physicians before taking such supplements $[42,43]$.

- Lastly, broccoli, Brussels sprouts, oranges and grapefruit, cherries, apples, and spinach that are rich in calcium D-glucarate (the calcium component of D-glucaric acid, which is contained in these foods) can help rid the body of toxins and the body eliminate excess estrogen [44]. For endometriosis, calcium D-glucarate may relieve symptoms caused by too much estrogen. The recommended dose is suggested between 500 to 1000 milligrams one to three times daily [45]

\section{DISCUSSION}

There is ample evidence in the literature concerning the beneficial action of a number of natural nutrients that can activate mechanisms for altering and/or improving human health. However, since the definition of functional foods has not universally been regulated [14] and the effects obtained by the aforementioned "bioactive compounds" supplied either as commercial pharmaceutical preparations or taken up by simply eating "functional foods" seem advantageous and often miraculous in certain conditions, caution is advised when it comes to vitamin use. Vitamin-related toxicity is frequently observed; if taken incorrectly or in excess, vitamin uptake may be a potential health hazard [46]. Almost 60,000 instances of vitamin toxicity are reported annually to the United States (US) poison control centers. Due to their ability to accumulate in the body, fat-soluble vitamins have a higher potential for toxicity than water-soluble vitamins do. Iron-containing vitamins are the most toxic, especially in pediatric acute ingestions. Potential risks of inappropriate vitamin and supplement regimes include an increased risk of coronary heart disease, hypertension, thrombophlebitis, peripheral neuropathy, ataxia, neurological effects, liver toxicity, congenital abnormalities, gouty arthritis, jaundice, kidney stones, diarrhea and even spontaneous abortion [47].

\section{CONCLUSION}

As many patients show intolerance and/or allergic reactions against a number of chemical pharmaceutical compositions or their excipients, efforts focus on the use of naturally occurring substitute target-specific treatments with fewer side-effects. Under this prism, the up-to-date incurable condition of endometriosis needs to be meticulously explored since a remedial action may be achieved by targeting the MESC via the fortification of the immune system using bioactive compounds. Although there are reports analyzing possible operating modes of a load of naturally occurring nutrients [48], a 2018 review gives insight on the anti-inflammatory activities of certain medicinal plants from Africa [49], thereby opening a new window for the examination of immune parameters that, as in the present case, may be of importance. However, since there is a long way to go for the full exploration of all potential pathways a functional food may trigger or follow, it is imperative to undertake studies for investigating the action of these bioactive substances, particularly on the induction of the appropriate immune conditions that may, can, or will fortify the organism immunologically to intercept MESC at early stages for reversing the burden of the disease. 
Abbreviations: Mesenchymal endometriotic stem cell, MESC; Endometriotic stromal cells, ESCs; Prostaglandin E, PGE; Coenzyme Q10, CoQ10; Diindolylmethane, DIM; T regulatory cells, Tregs; United States, US

Competing interests: The authors declare no conflict of interest in regard to this study.

Authors' contributions: Alexandros Vlachos, MD, performed the literature search and wrote the first draft of the manuscript. Simon Vassiliadis, Ph.D., conceived the idea of this review, compiled the data and wrote the manuscript having the primary responsibility for the final content. Both authors have read, edited and approved the final manuscript.

Acknowledgements and funding: The present communication is part of the "International program for the eradication of endometriosis via the incapacitation/interception of the nonhematopoietic mesenchymal endometriotic stem cell using natural remedies having the potential to act through the fortification of the immune system" launched by the Association of Greek Immunology Graduates (AGIG) in 2011. This article, however, was not financially supported by any organization or funding agency.

\section{REFERENCES}

1. Giudice LC, Kao LC: Endometriosis. Lancet. 2004;364(9447):1789-99.

2. Carter JE: Combined hysteroscopic and laparoscopic findings in patients with chronicpelvic pain. Journal of the American Association of Gynecologic Laparoscopists.1994;2:43-7.

3. Summaria V, Speca S, Mirk P: Ovarian factor infertility. Rays. 1998;23:709-26.

4. Halis G, Arici A. Endometriosis and inflammation in infertility. Annals of the New York Academy of Sciences. 2004;1034:300-15.

5. Senturk L, Arici A: Immunology of endometriosis. Journal of Reproductive Immunology. 1998;43:67-83.

6. Vassiliadis S, Relakis K, Papageorgiou A, Athanassakis I: Endometriosis and infertility: A multi-cytokine imbalance versus ovulation, fertilization and early embryo development. Clinical and Developmental Immunology. 2005;12:125-9.

7. Furuya K, Murakami M, Makimura N, Matsuda H, Ikou K, Saito K, Kawakami Y, et al.: Immunological and endocrinological studies on lymphocyte subpopulation and medical treatment for infertility in patients with endometriosis. Molecular and Cellular Endocrinology.2003;202:195-9.

8. Vinatier D, Dufour P, Oosterlynck D: Immunological aspects of endometriosis. Human Reproduction Update. 1996;2:371-84.

9. Crisp TM, Clegg ED, Cooper RL, Wood WR, Anderson DG, Baetcke KR, Hoffmann JL, et al.: Environmental endocrine disruption: An effects' assessment and analysis. Environmental Health Perspectives. 1998;106(Suppl 1):11-56.

10. Stefansson H, Geirsson RT, Steinthorsdottir V, Jonsson H, Manolescu A, Kong, A, Ingadottir $\mathrm{G}$ et al.: Genetic factors contribute to the risk of developing endometriosis. Human Reproduction. 2002;17:555-9.

11. Du H, Taylor HS: Contribution of bone marrow-derived stem cells to endometrium and endometriosis. Stem Cells. 2007;25:2082-6. 
12. Vassiliadis S: Premature immunosenescence impairs immune surveillance allowing the endometriotic stem cell to migrate: The cytokine profile as a common denominator. Journal of Endometriosis (currently: Journal of Endometriosis and Pelvic Pain Disorders). 2010;2:7-18.

13. Vassiliadis S: Can incapacitation of the mesenchymal endometriotic stem cell via the fortification of the immune system lead to the eradication of endometriosis? Journal of Endometriosis (currently: Journal of Endometriosis and Pelvic Pain Disorders). 2011;3:1-7.

14. Martirosyan D, Miller E: Bioactive compounds: The key to functional foods. Bioactive Compounds in Health and Disease. 2018;8(7):36-9.

15. Martirosyan D, Pisarski K: Bioactive compounds: Their role in functional food and human health, classifications, and definitions. Bioactive Compounds and Cancer. Edited by Danik Martirosyan and Jin-Rong Zhou: San Diego: Food Science Publisher; 238-277.

16. Pauling L: Orthomolecular psychiatry. Varying the concentrations of substances normally present in the human body may control mental disease. Science. 1968;160(3825):265-71.

17. Braverman E: Orthomolecular medicine and megavitamin therapy: Future and philosophy. Orthomolecular Psychiatry. 1979;8(4):265-72.

18. Use of mega vitamins for serious diseases. [http://www.1cure4cancer.com/use-megavitamins-serious-diseases/]

19. Saul AW: Not taking supplements causes miscarriage, birthing problems, infant mortality. [http://orthomolecular.org/resources/omns/v10n06.shtml]

20. Herington JL, Glore DR, Lucas JA, Osteen KG, Bruner-Tran KL: Dietary fish oil supplementation inhibits formation of endometriosis-associated adhesions in a chimeric mouse model. Fertility and Sterility. 2013;99(2):543-50.e1.

21. Guney M, Oral B, Karahan N, Mungan T: Regression of endometrial explants in a rat model of endometriosis treated with melatonin. Fertility and Sterility. 2008;89(4):93442.

22. Abbas MA, Taha MO, Zihlif MA, Disi AM: $\beta$-caryophyllene causes regression of endometrial implants in a rat model of endometriosis without affecting fertility. European Journal of Pharmacology. 2013;702(1-3):12-9.

23. Dimitrieva N, Nagabukuro H, Resuehr D, Zhang G, McAllister SL, McGinty KA, Mackie K et al.: Endocannabinoid involvement in endometriosis. Pain. 2010;151(3):70310.

24. Kojima T, Tanaka T, Mori H: Chemoprevention of spontaneous endometrial cancer in female Donryu rats by dietary indole-3-carbinol. Cancer Research. 1994;54(6):1446-9.

25. Dalessandri KM, Firestone GL, Fitch MD, Bradlow HL, Bjeldanes LF: Pilot study: Effect of 3,3'-diindolylmethane supplements on urinary hormone metabolites in postmenopausal women with a history of early-stage breast cancer. Nutrition and Cancer. 2004;50(2):161-7.

26. Huang HY, Caballero B, Chang S, Alberg AJ, Semba RD, Schneyer CR, Wilson RF, et al.: The efficacy and safety of multivitamin and mineral supplement use to prevent cancer and chronic disease in adults: A systematic review for a National Institutes of Health state-of-the-science conference. Annals of Internal Medicine. 2006;145(5):372-85.

27. Kapoor P, Kapoor AK: Coenzyme Q10 - A novel molecule. Journal, Indian Academy of Clinical Medicine. 2013;14(1):37-45. 
28. Kraft TE, Parisotto D, Schempp C, Efferth T: Fighting cancer with red wine? Molecular mechanisms of resveratrol. Critical Reviews in Food Science and Nutrition. 2009;49(9):782-99.

29. Kolahdouz-Mohammadi R, Arablou T: Resveratrol and endometriosis: In vitro and animal studies and underlying mechanisms (Review). Biomedicine and Pharmacotherapy. 2017;91:220-28.

30. Bruner-Tran KL, Osteen KG, Taylor HS, Sokalska A, Haines K, Duleba AJ: Resveratrol inhibits development of experimental endometriosis in vivo and reduces endometrial stromal cell invasiveness in vitro. Biology of Reproduction. 2011;84(1):106-12.

31. Mendes da Silva D, Gross LA, Neto EPG, Lessey BA, Savaris RF: The use of resveratrol as an adjuvant treatment of pain in endometriosis: A randomized clinical trial. Journal of Endocrine Society. 2017;1(4):359-69.

32. Mansour G, Abdelrazik H, Sharma RK, Radwan E, Falcone T, Agarwal A: L-Carnitine supplementation reduces oocyte cytoskeleton damage and embryo apoptosis induced by incubation in peritoneal fluid from patients with endometriosis. Fertility and Sterility. 2009;91:2079-86.

33. Vassiliadis S, Athanassakis I: A "conditionally-essential" nutrient, L-Carnitine, as a primary suspect of endometriosis. Fertility and Sterility. 2011;95:2759-60.

34. FAO/WHO: Human vitamin and mineral requirements. Report of a joint FAO/WHO expert consultation (Bangkok, Thailand, 1998). 2001; Food and Nutritional Division, FAO, Rome.

35. FAO: Requirements of vitamin A, iron, folate and vitamin $B_{12}$. FAO Nutrition Series No. 23. 1988; Food and Agriculture Organization, Rome.

36. Levander OA: A global view of human selenium nutrition. Annual Review of Nutrition. 1987;7:227-50.

37. Shankar AH, Prasad AS: Zinc and immune function: The biological basis of altered resistance to infection. American Journal of Clinical Nutrition. 1998;68(suppl):447S$463 \mathrm{~S}$.

38. Hoorn RK, Flikweert JP, Westerink D: Vitamin B1, B2 and B6 deficiencies in geriatric patients, measured by co-enzyme stimulation of enzyme activities. Clinica Chimica Acta. 1975;61:151-62.

39. Elhusseini H, Elkafas H, Abdelaziz M, Halder S, Atabiekov I, Eziba N, Ismail N, et al.: Diet-induced vitamin D deficiency triggers inflammation and DNA damage profile in murine myometrium. International Journal of Women's Health. 2018;10:503-14.

40. Vassiliadis S: Immune and homeostatic surveillance are positively modulated in cancerlike and cancer conditions by a number of natural products. Functional Foods in Health and Disease. 2015;5:331-8.

41. Pool-Zobel BL, Bub A, Muller H, Wollowski I, Rechkemmer G: Consumption of vegetables reduces genetic damage in humans: First results of a human intervention trial with carotenoid-rich foods. Carcinogenesis. 1997;18:1847-50.

42. Fijan S: Microorganisms with claimed probiotic properties: An overview of recent literature. International Journal of Environmental Research and Public Health. 2014;11:4745-67.

43. Markowiak P, Slizewska K: Effects of probiotics, prebiotics, and synbiotics on human health. Nutrients. 2017;9:1021-51. 
44. Walaszek Z, Hanausek-Walaszek M, Minto JP, Webb TE: Dietary glucarate as antipromoter of 7,12-dimethylbenz[a]anthracene-induced mammary tumorigenesis. Carcinogenesis. 1986; 7:1463-6.

45. Zoltaszek R, Kowalczyk P, Kowalczyk MC, Hanausek M, Kilianska ZM, Slaga TJ, Walaszek Z: Dietary D-glucarate effects on the biomarkers of inflammation during early post-initiation stages of benzo[a]pyrene-induced lung tumorigenesis in $\mathrm{A} / \mathrm{J}$ mice. Oncology Letters. 2011;2(1):145-54.

46. Blair KA: Vitamin supplementation and megadoses. Nurse Practitioner. 1986;11(7):1926 and 31-36 (abstracts).

47. Vitamin toxicity: Practice essentials, pathophysiology and etiology [https:// emedicine.medscape.com/article/819426-overview]

48. Vassiliadis S, Vlachos A: Potential advantages of medicinal plants over pharmaceutical compositions in endometriosis through the fortification of the mesenchymal endometriotic stem cell. Academia Journal of Medicinal Plants. 2018;6(7):186-90.

49. Oguntibeju OO: Medicinal plants with anti-inflammatory activities from selected countries and regions of Africa. Journal of Inflammation Research. 2018;11:307-17 